\title{
Rational oder emotional - warum Ernährungsberatung oft nichts erreicht!
}

Der aktuelle Ernährungsbericht der Deutschen Gesellschaft für Ernährung e.V. zeigt, dass sich das Ernährungsverhalten der Bevölkerung trotz jahrzehntelanger Aufklärung noch immer nicht nachhaltig verbessert hat. Die Alkohol- sowie Fettaufnahme ist zu hoch und die Nahrungsfaserzufuhr ist zu gering. Die Cholesterinaufnahme der Frauen liegt im Normalbereich, während Männer noch zu viel Cholesterin aufnehmen. Die Menschen sind hierzulande zunehmend verwirrt wenn es um die Ernährung und das Essen geht. Anstatt die Botschaften der wissenschaftlich agierenden Ernährungsfachkräfte aufzunehmen und umzusetzen, dass beispielsweise Gemüse und Obst wichtig für eine gesundheitsförderliche Ernährungsweise sind, bleibt bei ihnen vielmehr die Nachricht haften,

Die Bevölkerung hat dass Paprika aus Spanien mehr Angst vor schadstoffbelastet ist. Die BeE-Nummern als vor völkerung hat mehr Angst vor Überernährung E-Nummern als vor der Überund Fehlernährung. Die Kluft zwischen den von Experten und der Bevölkerung eingeschätzten Ernährungsrisiken nimmt zu. Aber wer ist für diese Situation verantwortlich zu machen oder ist die Bevölkerung einfach nur somnambul?

Kritisch ist insbesondere die Widersprüchlichkeit von ernährungsmedizinischen und ernährungswissenschaftlichen Aussagen. Momentan sprechen wir zu selten mit einer Sprache und unsere Argumentation geht viel zu oft an den Menschen vorbei. Wir müssen akzeptieren, dass das Ernährungsverhalten vorwiegend emotional bestimmt ist. Es ist leichter, falsche Nachrichten wie "Süßstoff macht dick" als die für die Medien langweiligen Nachricht "Süßstoffe können bei der Gewichtsreduktion helfen« zu kommunizieren.

In der Diätetik muss in vielen Bereichen ein Paradigmenwechsel erfolgen, um die ernährungsmedizinischen Erkenntnisse an den Patienten heranzutragen. Es ist obsolet, einen Patienten mit dekompensierter Leberzirrhose grundsätzlich proteinarm oder einen Patienten mit Dyslipidämie fettarm zu ernähren. Vielmehr benötigt ein Leberzirrhotiker im Rahmen seiner individuellen Proteintoleranz eine relativ erhöhte Proteinzufuhr und gegebenenfalls zusätzlich verzweigtkettigte Aminosäuren. Ein Dyslipidämiker benötigt eine fettmodifizierte Kostform, die Lein-, Raps- oder Walnussöl einschließt, um zu einer optimierten Fettsäurezufuhr - insbesondere zum Aus- gleich des allgemein bestehenden Ungleichgewichtes von Omega-3-zu Omega-6-Fettsäuren - zu gewährleisten. In der diätetischen Therapie kommt dem Perillaöl im Zusammenspiel mit einer täglich adäquaten Zufuhr natürlicher Antioxidantien - nicht nur für Diabetiker eine große Bedeutung zu, um vor Endothelschäden und damit schließlich auch vor dem Myokardinfarkt schützen zu können.

Gerade in der diätetischen Therapie ist das therapeutische Team von zunehmender Bedeutung. Es bleibt zu hoffen, dass an der Universitätsklinik Aachen zum Wintersemester 2005 der berufsbegleitende Masterstudiengang Master of Nutrition installiert wird. Die Mitglieder des diättherapeutischen Teams müssen durch Fortbildung gleichberechtigt in Anordnung und Ausführung der therapeutischen Leistung Diätberatung werden. Gerade in der Ausbildung im Bereich Diätetik sind noch zu große Unterschiede oder extreme Lücken festzustellen.

Die Aufgabe des dritten Jahrtausends wird in der Bekämpfung der Überernährung liegen. In der diätetischen Adipositastherapie ist ein Paradigmenwechsel zu erwarten, der zu einer Neubewertung der Proteine führt. In jedem Falle werden Aminosäuren zur Prophylaxe und Therapie von KHK und Depressionen sowie Proteine überhaupt in Zukunft einen Schwerpunkt der Ernährungsmedizin einnehmen. Adipositas muss auch vermehrt als endokrinologische Störung und entzündliche Erkrankung wahrgenommen werden. Hier fehlt es an schlüssigen Konzepten, die neben der dauerhaften Ernährungsumstellung aus dem Dilemma des sitzenden Lebensstils zu einem bewegten Alltag hin führen. Bei der Übergewichtsbekämpfung spielen Nahrungsfasern eine bedeutende Rolle, denn abnehmen kann nur wer satt is(s)t. Sättigung fängt im Magen an und endet im Hunger-Sättigungszentrum. Ungeklärt ist noch immer, ob der richtige diätetische Weg von vielen kleinen Mahlzeiten hin zu drei sättigenden größeren Mahlzeiten läuft. Zunehmend müssen wir Ernährungsmärchen und -mythen entzaubern und den langen Weg von der rationalen Vermittlung hin zur partnerschaftlich emotionsorientierten und lebenslangen Begleitung beschreiten. Sonst müssen unsere Bemühungen scheitern und eine weitere unzureichende Veränderung des Ernährungsverhaltens im Ernährungsbericht 2008 ist schon heute vorauszusagen.

Sven-David Müller-Nothmann 